Portland State University

PDXScholar

7-1-1995

\title{
Generalized Beam Matrices: Gaussian Beam Propagation in Misaligned Complex Optical Systems
}

\author{
Anthony A. Tovar \\ Portland State University \\ Lee W. Casperson \\ Portland State University
}

Follow this and additional works at: https://pdxscholar.library.pdx.edu/ece_fac

Part of the Electrical and Computer Engineering Commons

Let us know how access to this document benefits you.

\section{Citation Details}

Anthony A. Tovar and Lee W. Casperson, "Generalized beam matrices: Gaussian beam propagation in misaligned complex optical systems," J. Opt. Soc. Am. A 12, 1522-1533 (1995).

This Article is brought to you for free and open access. It has been accepted for inclusion in Electrical and Computer Engineering Faculty Publications and Presentations by an authorized administrator of PDXScholar. Please contact us if we can make this document more accessible: pdxscholar@pdx.edu. 


\title{
Generalized beam matrices: Gaussian beam propagation in misalligned complex optical systems
}

\author{
Anthony A. Tovar and Lee W. Casperson \\ Department of Electrical Engineering, Portland State University, Portland, Oregon 97207-0751
}

Received October 4, 1994; revised manuscript received February 2, 1995; accepted February 8, 1995

\begin{abstract}
A novel $3 \times 3$ transfer-matrix method is developed to propagate off-axis Gaussian beams in astigmatic optical systems that may include tilted, displaced, or curved optical elements. Unlike in a previous generalized ray matrix formalism, optical elements that possess gain or loss such as Gaussian apertures, complex lenslike media, and amplifiers are included; and a new beam transformation is found. In addition, a novel exponential variable-reflectivity mirror, which displaces a Gaussian beam without changing its spot size, and a complex prismlike medium are introduced.
\end{abstract}

\section{INTRODUCTION}

The beginning of paraxial ray theory is often ascribed to the German mathematician and physicist Karl Friedrich Gauss (1777-1855), who wrote down in his classic 1840 memoir Dioptrische Untersuchungen two linear simultaneous equations whereby the height and the angle of an output ray are linked to the corresponding input quantities. ${ }^{1}$ However, English mathematician Arthur Cayley (1821-1895) did not invent modern matrix theory until 1858. ${ }^{2}$ Paraxial ray matrix concepts developed slowly and were popularized only with the advent of the laser. $^{3}$ These matrices were subsequently generalized to account for misalignment $t^{4}$ and axis curvature ${ }^{5}$ of optical elements. This theory involves the use of $3 \times 3$ matrices in which only six of the matrix elements are nontrivial. A summary of these generalized ray matrices is given in Ref. 6.

Meanwhile, Gaussian beam theory was popularized by Kogelnik, who showed that, like paraxial light rays, Gaussian beams could be traced through a wide variety of aligned optical systems by simple $2 \times 2$ matrix multiplication. ${ }^{7}$ Furthermore, Kogelnik's beam matrix representation for a given optical element was the same as the corresponding ray matrix representation. It was then suggested that the center of a Gaussian beam travels along the same trajectory as that of a light ray. ${ }^{8}$ The subsequent proof identified the restriction that the optical element or system must be lossless. ${ }^{9}$ However, lossless optical systems are represented only by beam matrices that are real. For these systems the $3 \times 3$ generalized ray matrices may be used to propagate the displacement and the slope of the amplitude center of Gaussian beams in tilted optical systems.

A notational difficulty arose when the Gaussian beam theory was applied to optical systems that include optical elements with gain or loss. Such optical elements are represented by complex-valued beam matrices. These elements, such as Gaussian-profiled apertures and amplifiers with a radial gain profile, are often crucial to determining even the qualitative mode structure of lasers.
In optical systems, Gaussian beam matrices are thus distinct from ray matrices, as the former are complex valued and the latter are ineffectual since there is no simple interpretation for complex-valued position and slope of a light ray. To overcome this, a beam transformation was found so that the center of a Gaussian beam may be traced through a complex (i.e., nonlossless) optical system. ${ }^{10}$ Similar transformations have also been found to trace axial ${ }^{11}$ and off-axis ${ }^{12}$ polynomial-Gaussian beams in complex optical systems. However, these transformations are appropriate only for aligned systems, and the complex-valued $2 \times 2$ Gaussian beam matrices cannot be extended as complex-valued $3 \times 3$ ray matrices to account for misaligned or curved optical elements that have gain or loss. With the lack of an existing transfer-matrix method to propagate Gaussian beams in misaligned complex optical systems, one must resort to system analysis on an ad hoc basis. ${ }^{13,14}$ Thus the intent of this paper is to identify a novel $3 \times 3$ matrix method that enables one to propagate Gaussian beams in optical systems that have both gain (or loss) and misalignments. Though the form of these matrices is different from that of the purely real generalized ray matrices, there are still only six independent matrix elements.

In Section 2 Heaviside's differential form of Maxwell's equations is solved for a curved complex lenslike medium. The solutions may be put into a form that suggests a transfer-matrix approach. This matrix approach is considered in Section 3, and it is found that a large number of optical elements fit within the framework of this generalized beam matrix theory. In particular, the complex lenslike medium, the Gaussian aperture, the spherical boundary, the thin lens, and the spherical mirror are generalized so that they may be tilted, misaligned, or curved. In addition, the generalized beam matrix is found for a new prismlike medium, tilted boundary, a tilted mirror, a misaligned $A B C D$ system, and an axis transformation. Of particular interest is the exponential apodized aperture, which displaces a Gaussian beam without changing its spot size. The results are summarized in Section 4. 


\section{GENERALIZED BEAM MATRIX FOR CURVED COMPLEX LENSLIKE MEDIA}

Complex lenslike media are materials in which the gain and/or the refractive index varies quadratically in the transverse direction. It is the purpose of this section to derive from first principles Gaussian beam propagation in these lenslike media, which are allowed to be curved. The solution is put into a new matrix form.

A usual and appropriate starting point for a Gaussian beam derivation is the Heaviside form of Maxwell's equations. Typically these Maxwell-Heaviside equations are combined to form a wave equation with the material polarization as a forcing function. This polarization may be obtained from the density-matrix approach, which suggests that for simple two-level atoms the material polarization has components both in temporal phase and in quadrature with an assumed harmonic driving field. This can be rigorously accounted for by establishing a time-independent Helmholtz equation involving a complex permittivity $\epsilon^{\prime}$. For this study the complex permittivity will be recast as a complex spatially varying propagation constant $k$, where $k^{2} \equiv \omega^{2} \mu \epsilon^{\prime}$. The real constants $\omega$ and $\mu$ are the angular frequency of the harmonically varying field and the material permeability, respectively. With these constraints the resulting differential equation is

$$
\nabla^{2} \overline{E^{\prime}}+k^{2} \overline{E^{\prime}}=-2 \nabla\left(\frac{\nabla k}{k} \cdot \overline{E^{\prime}}\right) .
$$

The primes are a reminder that the harmonic time dependence $\exp (i \omega t)$ has been factored out of the real electric field. The complex vector $\overline{E^{\prime}}$ can be separated into scalar components, and then Eq. (1) would represent three complex equations in three unknowns for a given complex spatially dependent propagation constant. These equations are coupled, and for rectangular field components the right-hand side of Eq. (1) is the only coupling term. For simplicity, this coupling term is approximated to be negligible compared with either of the two remaining terms through the scalar approximation. ${ }^{15-18}$ This approximation has greatest validity for the two dominant transverse components. ${ }^{9}$ However, the longitudinal component of the electric field can be obtained from, for example, Gauss's law for the electric field. Similarly, the magnetic field can be obtained from the electric field by means of Faraday's law. It should be emphasized that if the complex propagation constant is really constant (i.e., space independent), then the coupling term is identically zero and no approximation has been made. In either case the equations become uncoupled and can be solved separately. In this scalar approximation it is therefore possible to define a propagation constant that is different for each of the two rectangular field components, and this is a common way to account for media anisotropy. ${ }^{19}$

Even with the right-hand side of Eq. (1) neglected, the resulting equation is not trivial. However, in many cases the light beam is nearly a plane wave. Hence a plane wave traveling in the positive $z$ direction is factored out:

$$
\begin{aligned}
\overline{E^{\prime}}(x, y, z)= & \psi(x, y, z) \exp \left[-i \int_{0}^{z} k_{0}\left(z^{\prime}\right) \mathrm{d} z^{\prime}\right] \\
& \times\left[(\cos \chi) \overline{i_{x}}+(\sin \chi) \overline{i_{y}}\right]
\end{aligned}
$$

where $\chi$ is the angle of the linearly polarized field from the $x$ axis. With this substitution Eq. (1) can be rewritten in the scalar approximation as

$$
\begin{array}{rl}
\frac{\partial^{2} \psi}{\partial x^{2}}+\frac{\partial^{2} \psi}{\partial y^{2}}-2 & 2 k_{0} \frac{\partial \psi}{\partial z} \\
+ & {\left[k^{2}(x, y, z)-k_{0}^{2}-i \frac{\mathrm{d} k_{0}}{\mathrm{~d} z}\right] \psi=0,}
\end{array}
$$

where the paraxial approximation

$$
\left|\frac{\partial}{\partial z}\left(\frac{\partial \psi}{\partial z}\right)\right| \ll\left|-2 i k_{0} \frac{\partial \psi}{\partial z}\right|
$$

has been made. The paraxial approximation is distinct from, though consistent with, the previous approximation. The scalar approximation is interpreted as requiring that the material properties not vary significantly in a wavelength of material, whereas the paraxial approximation requires that the complex field amplitude not have any important variation in the distance of a wavelength in the direction of propagation. A third approximation that is usually made is that there is negligible gain in the distance of a wavelength. However, such an approximation is unnecessary and is not in conflict with the two other approximations. ${ }^{20}$ Therefore no such approximation is made here.

For arbitrary variations of the complex propagation constant it is difficult to obtain exact analytic solutions to the paraxial wave equation (3). However, analytic solutions to Eq. (3) can be found under a wide variety of conditions if the propagation constant is Taylor series expanded in the transverse directions, keeping only constant, linear, and quadratic terms:

$$
\begin{aligned}
k^{2}(x, y, z)= & {\left[k_{0}(z)-1 / 2 k_{1 x}(z) x-1 / 2 k_{2 x}(z) x^{2}\right.} \\
& \left.-1 / 2 k_{1 y}(z) y-1 / 2 k_{2 y}(z) y^{2}\right]^{2} \\
\approx & k_{0}(z)\left[k_{0}(z)-k_{2 x}(z) x^{2}-k_{1 x}(z) x\right. \\
& \left.-k_{2 y}(z) y^{2}-k_{1 y}(z) y\right]
\end{aligned}
$$

Thus a slowly varying transverse profile approximation is also made. In a slightly more general analysis, twisting lenslike media may also be treated by the addition of a $k_{x y}(z) x y$ term in Eq. (5). ${ }^{9}$

It should be noted that the assumption of an ideal quadratic propagation constant can be valid only out to some finite radius in a realizable medium. For simplicity, it is assumed here that the propagating modes are confined completely within the quadratic-profile region. However, a quadratic is an excellent approximation near the center of any smoothly peaked function, including the Bessel radial dependence of the gain in a gas discharge, ${ }^{21}$ a Gaussian radial dependence of the gain that is due to optical pumping, or similar profiles that are due to spatial hole burning. ${ }^{22}$ The complex propagation constant [Eq. (5)] can be separated into its real and imaginary parts as 


$$
\begin{aligned}
k(x, y, z)= & {\left[\beta_{0}+\frac{\beta_{1 x}{ }^{2}}{8 \beta_{2 x}}+\frac{\beta_{1 y}{ }^{2}}{8 \beta_{2 y}}-\frac{\beta_{2 x}}{2}\left(x+\frac{\beta_{1 x}}{2 \beta_{2 x}}\right)^{2}\right.} \\
& \left.-\frac{\beta_{2 y}}{2}\left(y+\frac{\beta_{1 y}}{2 \beta_{2 y}}\right)^{2}\right] \\
& +i\left[\alpha_{0}+\frac{\alpha_{1 x}{ }^{2}}{8 \alpha_{2 x}}+\frac{\alpha_{1 y}{ }^{2}}{8 \alpha_{2 y}}-\frac{\alpha_{2 x}}{2}\left(x+\frac{\alpha_{1 x}}{2 \alpha_{2 x}}\right)^{2}\right. \\
& \left.-\frac{\alpha_{2 y}}{2}\left(y+\frac{\alpha_{1 y}}{2 \alpha_{2 y}}\right)^{2}\right]
\end{aligned}
$$

where $\beta$ and $\alpha$ are the real and imaginary parts of the complex propagation constant, respectively. It can readily be seen that the effect of the linear terms $k_{1 x}$ and $k_{1 y}$ is to displace the quadratic medium from the $z$ coordinate axis. For each transverse axis it is sensible to define the displacements of the index of refraction axis, $d_{x \beta}, d_{y \beta}$, and the displacements of the gain axis, $d_{x \alpha}, d_{y \alpha}$, as

$$
\begin{aligned}
& d_{x \beta}(z)=-\frac{\beta_{1 x}(z)}{2 \beta_{2 x}(z)}, \\
& d_{y \beta}(z)=-\frac{\beta_{1 y}(z)}{2 \beta_{2 y}(z)}, \\
& d_{x \alpha}(z)=-\frac{\alpha_{1 x}(z)}{2 \alpha_{2 x}(z)}, \\
& d_{y \alpha}(z)=-\frac{\alpha_{1 x}(z)}{2 \alpha_{2 y}(z)} .
\end{aligned}
$$

As may be seen from Eq. (7), along an axis defined by the index of refraction $\left(x=d_{x \beta}, y=d_{y \beta}\right)$ or gain $\left(x=d_{x \alpha}, y=\right.$ $d_{y \alpha}$ ) of the optical medium, the effective axial propagation is different from one defined along the $z$ axis.

The goal now is to obtain exact solutions to Eq. (3) with the propagation constant [relation (6)] under a wide variety of conditions. Following Ref. 23, a Gaussian substitution of the form

$$
\begin{aligned}
\psi(x, y, z)= & \psi^{\prime}(x, y, z) \exp \left\{-i\left[1 / 2 Q_{x}(z) x^{2}+1 / 2 Q_{y}(z) y^{2}\right.\right. \\
& \left.\left.+S_{x}(z) x+S_{y}(z) y\right]\right\}
\end{aligned}
$$

yields a new equation,

$$
\begin{aligned}
\frac{\partial^{2} \psi^{\prime}}{\partial x^{2}}- & 2 i\left(S_{x}+Q_{x} x\right) \frac{\partial \psi^{\prime}}{\partial x}+\frac{\partial^{2} \psi^{\prime}}{\partial y^{2}} \\
& -2 i\left(S_{y}+Q_{y} y\right) \frac{\partial \psi^{\prime}}{\partial y}-\left({S_{x}}^{2}+S_{y}{ }^{2}\right) \psi^{\prime} \\
& -i\left(Q_{x}+Q_{y}\right) \psi^{\prime}-2 i k_{0} \frac{\partial \psi^{\prime}}{\partial z}-i \frac{d k_{0}}{d z} \psi^{\prime}=0
\end{aligned}
$$

when combined with the following choices for the $z$-dependent parameters:

$$
\begin{array}{r}
Q_{x}{ }^{2}+k_{0}(z) \frac{\mathrm{d} Q_{x}}{\mathrm{~d} z}+k_{0}(z) k_{2 x}(z)=0, \\
Q_{y}{ }^{2}+k_{0}(z) \frac{\mathrm{d} Q_{y}}{\mathrm{~d} z}+k_{0}(z) k_{2 y}(z)=0, \\
Q_{x} S_{x}+k_{0}(z) \frac{\mathrm{d} S_{x}}{\mathrm{~d} z}+\frac{k_{0}(z) k_{1 x}(z)}{2}=0, \\
Q_{y} S_{y}+k_{0}(z) \frac{\mathrm{d} S_{y}}{\mathrm{~d} z}+\frac{k_{0}(z) k_{1 y}(z)}{2}=0 .
\end{array}
$$

Equation (13) may be simplified substantially for fundamental-mode propagation. In particular, if $\psi^{\prime}(x, y, z)=\exp [-i P(z)]$, then the phase parameter equation is

$$
2 k_{0} \frac{\mathrm{d} P}{\mathrm{~d} z}+i\left(Q_{x}+Q_{y}\right)+\left(S_{x}{ }^{2}+S_{y}{ }^{2}\right)+i \frac{\mathrm{d} k_{0}}{\mathrm{~d} z}=0 .
$$

Equations (14) and (15) are beam parameter equations, and $Q_{x}$ and $Q_{y}$ are known as beam parameters. Each of these two equations is uncoupled and can be solved independently. Since the forms of Eqs. (14) and (16) for the beam's parameters in the $x$ direction are identical to those of Eqs. (15) and (17) for the beam's parameters in the $y$ direction, then for every equation written for the $x$ direction there is a corresponding equation for the $y$ direction. Thus, though only $x$-direction equations are written, astigmatism is accounted for. The Gaussian beam's $1 / e$ electric-field amplitude spot size and radius of phase front curvature at a flat plane are related to the beam parameter by the relation ${ }^{20}$

$$
Q_{x}(z)=\frac{\beta_{0}}{R_{x}(z)}-i \frac{2}{w_{x}^{2}(z)} .
$$

Equation (16) is sometimes called the displacement parameter equation, and $S_{x}$ is likewise known as the displacement parameter. Equation (16) is also uncoupled and can be solved independently once its corresponding beam parameter equation has been solved. The significance of the displacement parameter lies in the fact that if $d_{x a}$ is the displacement of the amplitude center of the beam in the $x$ direction and $d_{x p}$ is the displacement of the phase center of the beam in the $x$ direction, then, if we separate the argument of the exponential into real and imaginary parts and complete the squares in Eq. (12), it follows that

$$
\begin{aligned}
& d_{x a}(z)=-S_{x i}(z) / Q_{x i}(z), \\
& d_{x p}(z)=-S_{x r}(z) / Q_{x r}(z) .
\end{aligned}
$$

Here, and everywhere below, the $r$ and $i$ subscripts are meant to denote the real and imaginary parts of a complex function. Equations (20) and (21) may be inverted and combined into

$$
S_{x}(z)=-Q_{x r}(z) d_{x p}(z)-i Q_{x i}(z) d_{x a}(z) .
$$

As the displacement of the amplitude center represents the position of a Gaussian beam, the slope of a Gaussian beam can be obtained by taking a $z$ derivative of Eq. (20) and substituting Eqs. (14) and (16). In the $x$ direction the slope is found to be

$$
\begin{aligned}
\frac{\mathrm{d} d_{x a}}{\mathrm{~d} z}= & -\frac{\alpha_{2 x}}{Q_{x i}}\left(d_{x \alpha}-d_{x a}\right)+\left(\frac{1-\alpha_{\lambda} Q_{x r} / Q_{x i}}{1+\alpha_{\lambda}{ }^{2}}\right) \\
& \times\left(d_{x a}-d_{x p}\right) \frac{Q x r}{\beta_{0}} \\
= & \frac{\alpha_{2 x} w_{x}{ }^{2}}{2}\left(d_{x \alpha}-d_{x a}\right)+\left(\frac{1+\alpha_{\lambda} \pi n w_{x}^{2} / \lambda R_{x}}{1+\alpha_{\lambda}^{2}}\right) \\
& \times\left(\frac{d_{x a}-d_{x p}}{R_{x}}\right),
\end{aligned}
$$

where $\alpha_{\lambda} \equiv \alpha_{0} / \beta_{0}$ and the $z$ dependences have been suppressed for readability. Equation (23b) is written in this 
form to reduce to Eq. (14) of Ref. 24 when $\alpha_{2 x}=0$ and $\alpha_{\lambda}=0$. In this way the beam slope is related to the phase center of a Gaussian beam. Equation (22) can therefore be rewritten as

$$
\begin{aligned}
S_{x}= & -Q_{x r} d_{x a}+\beta_{0} \frac{1+\alpha_{\lambda}^{2}}{1-\alpha_{\lambda} Q_{x r} / Q_{x i}} \\
& \times\left[d_{x a}^{\prime}+\frac{\alpha_{2 x}}{Q_{x i}}\left(d_{x \alpha}-d_{x a}\right)\right]-i Q_{x i} d_{x a},
\end{aligned}
$$

where $d_{x a}^{\prime} \equiv \mathrm{d} d_{x a} / \mathrm{d} z$. Often, the input and output planes are chosen to be in a homogeneous dielectric medium (or free space), and in this case Eq. (24) reduces to

$$
S_{x}=-Q_{x} d_{x a}+\beta_{0} d_{x a}^{\prime} .
$$

The goal now is to solve Eqs (14)-(17) and express the results in terms of beam matrices. This is achieved by making the Ricatti substitution,

$$
\frac{Q_{x}(z)}{k_{0}(z)}=\frac{1}{u_{x}(z)} \frac{\mathrm{d} u_{x}}{\mathrm{~d} z},
$$

into Eqs. (14) and (16), which may be expressed as

$$
\begin{aligned}
\frac{\mathrm{d}}{\mathrm{d} z}\left[k_{0}(z) \frac{\mathrm{d} u_{x}}{\mathrm{~d} z}\right]+k_{2 x}(z) u_{x}(z) & =0, \\
\frac{\mathrm{d}}{\mathrm{d} z}\left[u_{x}(z) S_{x}(z)\right] & =-1 / 2 k_{1 x}(z) u_{x}(z) .
\end{aligned}
$$

As a linear homogeneous second-order differential equation, Eq. (27) has solutions that may be written as a linear combination of two independent functions:

$$
u_{x}(z)=A_{x}(z) u_{x}(0)+B_{x}(z) u_{x}^{\prime}(0) .
$$

The derivative may be written in a similar form:

$$
u_{x}^{\prime}(z)=C_{x}(z) u_{x}(0)+D_{x}(z) u_{x}^{\prime}(0) .
$$

If we take the $z$ derivative of Eq. (29), it follows that $C_{x}(z)=\mathrm{d} A_{x} / \mathrm{d} z$ and $D_{x}(z)=\mathrm{d} B_{x} / \mathrm{d} z$ in material media. If we substitute Eqs. (29) and (30) into Eq. (27) and use the fact that $A_{x}(z)$ and $B_{x}(z)$ are linearly independent, it follows that

$$
\begin{aligned}
& \frac{\mathrm{d}}{\mathrm{d} z}\left[k_{0}(z) C_{x}(z)\right]=-k_{2 x}(z) A_{x}(z), \\
& \frac{\mathrm{d}}{\mathrm{d} z}\left[k_{0}(z) D_{x}(z)\right]=-k_{2 x}(z) B_{x}(z) .
\end{aligned}
$$

These results may be used, for example, to show that the $z$ derivative of the product of $k_{0}(z)$ and the Wronskian, $A_{x}(z) D_{x}(z)-B_{x}(z) C_{x}(z)$, is zero, from which it follows that

$$
A_{x}(z) D_{x}(z)-B_{x}(z) C_{x}(z)=\frac{k_{0}(0)}{k_{0}(z)},
$$

where $k_{0}(0)$ and $k_{0}(z)$ are the axial complex propagation constants at the input plane and the output plane, respectively.

Equation (28) may be integrated directly, and from Eq. (29) it can be seen that

$$
\begin{aligned}
u_{x}(z) S_{x}(z)= & u_{x}(0) S_{x}(0)-\left[\frac{1}{2} \int_{0}^{z} k_{1 x}\left(z^{\prime}\right) A_{x}\left(z^{\prime}\right) \mathrm{d} z^{\prime}\right] u_{x}(0) \\
& -\left[\frac{1}{2} \int_{0}^{z} k_{1 x}\left(z^{\prime}\right) B_{x}\left(z^{\prime}\right) \mathrm{d} z^{\prime}\right] u_{x}^{\prime}(0)
\end{aligned}
$$

If we define

$$
\begin{aligned}
& G(z) \equiv-\frac{1}{2} \int_{0}^{z} k_{1 x}\left(z^{\prime}\right) A\left(z^{\prime}\right) \mathrm{d} z^{\prime}, \\
& H(z) \equiv-\frac{1}{2} \int_{0}^{z} k_{1 x}\left(z^{\prime}\right) B\left(z^{\prime}\right) \mathrm{d} z^{\prime}
\end{aligned}
$$

then Eqs. (29), (30), and (34) can be written in a new generalized beam matrix form:

$$
\begin{aligned}
\left(\begin{array}{c}
u_{x}(z) \\
{\left[Q_{x}(z) / k_{0}(z)\right] u_{x}(z)} \\
S_{x}(z) u_{x}(z)
\end{array}\right)= & {\left[\begin{array}{ccc}
A_{x}(z) & B_{x}(z) & 0 \\
C_{x}(z) & D_{x}(z) & 0 \\
G_{x}(z) & H_{x}(z) & 1
\end{array}\right] } \\
& \times\left(\begin{array}{c}
u_{x}(0) \\
{\left[Q_{x}(0) / k_{0}(0)\right] u_{x}(0)} \\
S_{x}(0) u_{x}(0)
\end{array}\right),
\end{aligned}
$$

where Eq. (26) has also been used. In SI units the dimension of $B_{x}(z)$ is meters, the dimensions of $C_{x}(z)$ and $G_{x}(z)$ are inverse meters, and $A_{x}(z), D_{x}(z)$, and $H_{x}(z)$ are dimensionless.

The three rows of matrix equation (37) represent three equations. It follows, by dividing the second equation by the first, that

$$
\frac{Q_{x}(z)}{k_{0}(z)}=\frac{C_{x}(z)+D_{x}(z) Q_{x}(0) / k_{0}(0)}{A_{x}(z)+B_{x}(z) Q_{x}(0) / k_{0}(0)} .
$$

This is the Kogelnik transformation. ${ }^{7}$ The beam parameter $Q_{x}$ is related to the light beam's spot size and radius of curvature through Eq. (19). In a similar manner the third equation divided by the first equation in Eq. (37) yields a new law analogous to the Kogelnik transformation:

$$
\begin{aligned}
S_{x}(z)= & \frac{S_{x}(0)}{A_{x}(z)+B_{x}(z) Q_{x}(0) / k_{0}(0)} \\
& +\frac{G_{x}(z)+H_{x}(z) Q_{x}(0) / k_{0}(0)}{A_{x}(z)+B_{x}(z) Q_{x}(0) / k_{0}(0)} .
\end{aligned}
$$

For aligned optical systems, $G_{x}=0$ and $H_{x}=0$ and Eq. (39) reduces to a transformation derived previously. ${ }^{10}$ In Appendix A it is shown that when the generalized beam matrix elements are purely real (i.e., for lossless optical systems), the propagation characteristics may be described in terms of a generalized ray matrix. Once the generalized beam matrix is known, the procedure to propagate a Gaussian beam's position, slope, spot size, and phase front curvature involves the use of the intermediate complex parameters $Q_{x}$ and $S_{x}$, which may be obtained from Eqs. (19) and (24), respectively. The output beam parameter, $Q_{x}(z)$, and the output displacement parameter, $S_{x}(z)$, may be obtained from Eqs. (38) and (39). The Gaussian beam's output parameters of interest may then be obtained from Eqs. (19) and (24). 
As a specific example of a generalized beam matrix, a complex lenslike medium with $k_{0}(z)=k_{0}$ and $k_{2 x}(z)=k_{2 x}$ is considered. In this case Eq. (27) is easily solved, and the corresponding generalized beam matrix is where the 1 and 2 subscripts represent the input and output parameters of the optical element and system, respectively. As solutions to the differential equations discussed in Section 2 require, the bottom, rightmost ele-

$T_{\text {complex lenslike medium }}=\left[\begin{array}{ccc}\cos \left(\gamma_{x} z\right) & \gamma_{x}^{-1} \sin \left(\gamma_{x} z\right) & 0 \\ -\gamma_{x} \sin \left(\gamma_{x} z\right) & \cos \left(\gamma_{x} z\right) & 0 \\ -\frac{1}{2} \int_{0}^{z} k_{1 x}\left(z^{\prime}\right) \cos \left(\gamma_{x} z^{\prime}\right) \mathrm{d} z^{\prime} & -\frac{1}{2} \gamma_{x}^{-1} \int_{0}^{z} k_{1 x}\left(z^{\prime}\right) \sin \left(\gamma_{x} z^{\prime}\right) \mathrm{d} z^{\prime} & 1\end{array}\right]$,

where Eqs. (35) and (36) have been used, as has the definition

$$
\gamma_{x} \equiv\left(\frac{k_{2 x}}{k_{0}}\right)^{1 / 2}
$$

For $z$-independent $k_{0}$ and $k_{2 x}$, the $G_{x}(z)$ and $H_{x}(z)$ integrations in Eq. (40) may be performed for arbitrarily curved media by Taylor series expanding $k_{1 x}(z)$ and applying successive integration by parts and noting Eqs. (31) and (32). Though this procedure to propagate an off-axis Gaussian beam in a curved complex lenslike medium is straightforward, the usefulness of the generalized beam matrices is amplified by its application to more complicated systems of optical elements, which is considered in Section 3.

\section{GENERALIZED BEAM MATRICES FOR OPTICAL SYSTEMS}

In Section 2 generalized beam matrices were introduced, and the generalized beam matrix for a curved complex lenslike medium was identified. The purpose of this section is to identify generalized beam matrices for several other optical elements and apply the matrix method to optical systems. To encourage analogies, we divide optical elements into three classes: nonprofiled, linearly profiled, and quadratically profiled. Nonprofiled optical elements such as homogeneous media possess no phase variation or amplitude transmission variation in either of the transverse directions. Similarly, linearly profiled optical elements are linear in the transverse variations of their complex propagation constants. Prisms and tilted mirrors are familiar examples of linearly profiled optics. In the same manner lenses and spherical mirrors (which are approximately parabolic) are examples of quadratically profiled optical elements.

For a system of optical elements it is sensible to designate the various reference planes numerically. In particular, the generalized beam matrix may be written as

$$
\left(\begin{array}{c}
u_{x 2} \\
\left(Q_{x 2} / k_{02}\right) u_{x 2} \\
S_{x 2} u_{x 2}
\end{array}\right)=\left[\begin{array}{ccc}
A_{x} & B_{x} & 0 \\
C_{x} & D_{x} & 0 \\
G_{x} & H_{x} & 1
\end{array}\right]\left(\begin{array}{c}
u_{x 1} \\
\left(Q_{x 1} / k_{01}\right) u_{x 1} \\
S_{x 1} u_{x 1}
\end{array}\right)
$$

ment of the generalized beam matrix is unity. However, since the observable beam properties exist only as ratios of $u_{x}, Q_{x} k_{0}^{-1} u_{x}$, and $S_{x} u_{x}$, these observables are unchanged if the beam matrix is multiplied by any nonzero $z$-dependent function. Thus, if the bottom, rightmost element is initially nonzero, the generalized beam matrix may be scaled to make it unity. However, there may exist certain peculiar matrices in which the bottom, rightmost element is zero. In this case the output position and slope of the Gaussian beam would be independent of the input position and slope. Though it is straightforward to extend the results below to account for this effect, this possibility is excluded from further consideration here.

As in Section 2, the Kogelnik transformation is obtained by dividing the second row of Eq. (42) by the first row:

$$
\frac{Q_{x 2}}{k_{02}}=\frac{C_{x}+D_{x} Q_{x 1} / k_{01}}{A_{x}+B_{x} Q_{x 1} / k_{01}}
$$

The displacement transformation is obtained by dividing the third row of Eq. (42) by the first row:

$$
S_{x 2}=\frac{S_{x 1}}{A_{x}+B_{x} Q_{x 1} / k_{01}}+\frac{G_{x}+H_{x} Q_{x 1} / k_{01}}{A_{x}+B_{x} Q_{x 1} / k_{01}} .
$$

An alternative, but redundant, transformation may be obtained either by dividing the second row of Eq. (42) by the third row or by dividing Eq. (44) by Eq. (43).

As is typical in transfer-matrix methods, system analysis merely consists of matrix multiplication of the individual elements in reverse order. Though the procedure for obtaining the generalized beam matrix for any medium described by Eq. (7) has been shown above, the only generalized beam matrix derived thus far represents a complex lenslike medium. Since the value of $\gamma_{x}$ and the variation of $k_{1 x}$ in Eq. (40) are arbitrary, it is straightforward to analyze optical systems that consist only of piecewise continuous curved complex lenslike media with these different properties. In the limit of small $\gamma_{x}$, an arbitrarily curved complex lenslike medium is a special case of a complex prismlike medium. For arbitrary variations of $k_{0}(z)$ and $k_{1 x}(z)$ Eqs. (27), (35), and (36) are simply integrals, since $k_{2 x}(z)=0$, and the matrix for a complex prismlike medium is

$$
T_{\text {complex prismlike medium }}=\left[\begin{array}{ccc}
1 & \int_{0}^{d} k_{0}(0) k_{0}{ }^{-1}(z) \mathrm{d} z & 0 \\
0 & k_{0}(0) k_{0}^{-1}(z) & 0 \\
-\frac{1}{2} \int_{0}^{d} k_{1 x}(z) \mathrm{d} z & -\frac{1}{2} \int_{0}^{d} k_{1 x}(z) \int_{0}^{z} k_{0}(0) k_{0}^{-1}\left(z^{\prime}\right) \mathrm{d} z^{\prime} \mathrm{d} z & 1
\end{array}\right] \text {. }
$$


Table 1. Generalized Beam Matrices for Nonprofiled Elements

$\mathrm{D}$ Generalized Beam Matrix

\section{Optical Medium}

Homogeneous medium

Aligned mirror

Aligned boundary

Retroreflector

Axial medium

Axis transformation

Misaligned $A B C D$
Tangential Plane $(x)$ $\left[\begin{array}{lll}1 & d & 0 \\ 0 & 1 & 0 \\ 0 & 0 & 1\end{array}\right]$

$\left[\begin{array}{lll}1 & 0 & 0 \\ 0 & 1 & 0 \\ 0 & 0 & 1\end{array}\right]$

$\left[\begin{array}{ccc}1 & 0 & 0 \\ 0 & k_{01} / k_{02} & 0 \\ 0 & 0 & 1\end{array}\right]$

$\left[\begin{array}{rrr}-1 & 0 & 0 \\ 0 & -1 & 0 \\ 0 & 0 & 1\end{array}\right]$

$\left[\begin{array}{ccc}1 & k_{0}(0) \int_{0}^{d} k_{0}^{-1}(z) \mathrm{d} z & 0 \\ 0 & k_{0}(0) k_{0}^{-1}(d) & 0 \\ 0 & 0 & 1\end{array}\right]$

$\left[\begin{array}{ccc}1 & 0 & 0 \\ 0 & 1 & 0 \\ \tan \theta_{x} & -x_{0} & 1\end{array}\right]$

$\left[\begin{array}{lll}A_{x} & B_{x} & 0 \\ C_{x} & D_{x} & 0 \\ G_{x} & H_{x} & 1\end{array}\right]$
$G_{x}=\beta_{0}\left[\left(A_{x}-1\right) \tan \theta_{x}-C_{x}\left(x_{0}+L \sin \theta_{x}\right)\right]$

$H_{x}=\beta_{0}\left[\left(1-D_{x}\right) x_{0}+B_{x} \tan \theta_{x}-D_{x} L \sin \theta_{x}\right]$
Sagittal Plane $(y)$

$\left[\begin{array}{lll}1 & d & 0 \\ 0 & 1 & 0 \\ 0 & 0 & 1\end{array}\right]$

$\left[\begin{array}{lll}1 & 0 & 0 \\ 0 & 1 & 0 \\ 0 & 0 & 1\end{array}\right]$

$\left[\begin{array}{ccc}1 & 0 & 0 \\ 0 & k_{01} / k_{02} & 0 \\ 0 & 0 & 1\end{array}\right]$

$\left[\begin{array}{rrr}-1 & 0 & 0 \\ 0 & -1 & 0 \\ 0 & 0 & 1\end{array}\right]$

$\left[\begin{array}{ccc}1 & k_{0}(0) \int_{0}^{d} k_{0}^{-1}(z) \mathrm{d} z & 0 \\ 0 & k_{0}(0) k_{0}^{-1}(d) & 0 \\ 0 & 0 & 1\end{array}\right]$

$\left[\begin{array}{ccc}1 & 0 & 0 \\ 0 & 1 & 0 \\ \tan \theta_{y} & -y_{0} & 1\end{array}\right]$

$\left[\begin{array}{lll}A_{y} & B_{y} & 0 \\ C_{y} & D_{y} & 0 \\ G_{y} & H_{y} & 1\end{array}\right]$

$G_{y}=\beta_{0}\left[\left(A_{y}-1\right) \tan \theta_{y}-C_{y}\left(y_{0}+L \sin \theta_{y}\right)\right]$

$H_{y}=\beta_{0}\left[\left(1-D_{y}\right) y_{0}+B_{y} \tan \theta_{y}-D_{y} L \sin \theta_{y}\right]$
When $k_{1 x}(z)=0$ and $k_{0}$ is constant, Eq. (45) reduces to the generalized beam matrix for a homogeneous medium.

\section{A. Axis Transformation}

The matrix representations for several nonprofiled optical elements are well known and are listed in the first five rows of Table 1. However, the boundary matrix and the axial medium matrix are generalized here so that the low nonsaturating gain per wavelength approximation is not made. ${ }^{20}$ Small tilts of an optical element or system of elements may be accounted for by the use of a $z$-axis transformation (see, for example, Ref. 6). Thus the purpose of this subsection is to derive the generalized beam matrix for two optical elements. The first axis transformation changes the position and the slope of the optic axis. The second beam matrix is for an aligned optical system that is tilted with respect to the optic axis.

The axis transformation matrix represents one of a class of thin optical elements. In optical systems in which the media at the input and the output are identical, many of these thin optical elements have the property that $A=D=1$ and $B=0$. Therefore, for any such optical element, the displacement parameter transformation [Eq. (44)] reduces to

$$
S_{x 2}=S_{x 1}+G_{x}+H_{x} \frac{Q_{x 1}}{k_{01}}
$$

and the Kogelnik transformation [Eq. (43)] reduces to

$$
\frac{Q_{x 2}}{k_{02}}=C_{x}+\frac{Q_{x 1}}{k_{01}} .
$$

To examine the effects of this transformation on the position and the slope of a Gaussian beam, we substitute Eqs. (22) and (47) into Eq. (46) and separate the result into real and imaginary parts. Without loss of generality, it is assumed that the media at the input and the output of the system are free space. It then follows from Eqs. (20) and (21) that

$\begin{aligned} d_{x a 2} & =\frac{Q_{x 1 i} d_{x a 1}-G_{x i}-H_{x i} Q_{x 1 r} / \beta_{0}-H_{x r} Q_{x 1 i} / \beta_{0}}{Q_{x 1 i}+\beta_{0} C_{x i}}, \\ d_{x p 2} & =\frac{Q_{x 1 r} d_{x p 1}-G_{x r}-H_{x r} Q_{x 1 r} / \beta_{0}+H_{x i} Q_{x 1 i} / \beta_{0}}{Q_{x 1 r}+\beta_{0} C_{x r}},\end{aligned}$

where, as in Section 2, the rand $i$ subscripts represent the real and imaginary parts of the complex quantity, respectively. The phase and amplitude displacements are related to the beam slope from Eqs. (23). When the input and output media are free space, Eqs. (23) reduce to

$$
d_{x a}^{\prime}=\left(d_{x a}-d_{x p}\right) \frac{Q_{x r}}{\beta_{0}} .
$$

Combining Eqs. (47)-(50) yields 


$$
\begin{aligned}
d_{x a 2}^{\prime}= & \left(\frac{C_{x r} Q_{x 1 i}-C_{x i} Q_{x 1 r}}{Q_{x 1 i}+\beta_{0} C_{x i}}\right)\left(d_{x a 1}-H_{x r} / \beta_{0}\right)+d_{x a 1}^{\prime} \\
& +\frac{G_{x r}-H_{x i} Q_{x 1 i} / \beta_{0}}{\beta_{0}}-\left(\frac{Q_{x 1 r}+\beta_{0} C_{x r}}{Q_{x 1 i}+\beta_{0} C_{x i}}\right) \\
& \times \frac{G_{x i}+H_{x i} Q_{x 1 r} / \beta_{0}}{\beta_{0}} .
\end{aligned}
$$

This equation, used with Eq. (48), is useful for determining the effects of a thin element on the position and the slope of a Gaussian beam. As an example, the Gaussian aperture is considered. For an undisplaced aligned Gaussian aperture, $G_{x}=0, H_{x}=0$, and $C_{x}=$ $-2 i / \beta_{0} w_{g a, x}{ }^{2}$, where $w_{g a, x}$ is the width of the aperture. In this case Eqs. (48) and (51) reduce to

$$
\begin{aligned}
& d_{x a 2}=\frac{d_{x a 1}}{1+\left(w_{x 1} / w_{g a, x}\right)^{2}}, \\
& d_{x a 2}^{\prime}=-\frac{R_{x 1}{ }^{-1}\left(w_{x 1} / w_{g a, x}\right)^{2}}{1+\left(w_{x 1} / w_{g a, x}\right)^{2}} d_{x a 1}+d_{x a 1}^{\prime},
\end{aligned}
$$

$$
T_{\text {axis transformation, } x}=\left[\begin{array}{ccc}
1 & 0 & 0 \\
0 & 1 & 0 \\
\beta_{0} \tan \theta_{x} & -\beta_{0} x_{0} & 1
\end{array}\right] .
$$

Since the matrix elements are real, this generalized beam matrix may also be derived by use of Eqs. (56) and (57) together with the generalized ray matrix conversion formulas (A13) and (A14) in Appendix A. However, it should be emphasized that the ray matrix techniques may not be used for complex-valued matrices and could not be used, for example, to derive Eqs. (52) and (53).

The axis transformation matrix (58) may be used to obtain the generalized Gaussian beam matrix for a tilted optical system that would otherwise be representable by an ordinary complex $A B C D$ matrix. The methodology consists of postmultiplying the $A B C D$ matrix to tilt the optic axis and premultiplying the $A B C D$ matrix to transform the optic axis back to its original position and slope. ${ }^{6}$ The difference of the position of the axis at the output from that at the input is $L \sin \theta_{x}$, where $L$ is the length and $\theta_{x}$ is the tilt angle of the $A B C D$ system. The matrix for an aligned system with a global tilt angle of $\theta$ is

$$
\begin{aligned}
T_{\operatorname{tilted} A B C D, x} & =\left[\begin{array}{ccc}
1 & 0 & 0 \\
0 & 1 & 0 \\
\beta_{0} \tan \theta_{x} & -\beta_{0}\left(x_{0}+L \sin \theta_{x}\right) & 1
\end{array}\right]\left[\begin{array}{ccc}
A_{x} & B_{x} & 0 \\
C_{x} & D_{x} & 0 \\
0 & 0 & 1
\end{array}\right]\left[\begin{array}{ccc}
1 & 0 & 0 \\
0 & 1 & 0 \\
-\beta_{0} \tan \theta_{x} & \beta_{0} x_{0} & 1
\end{array}\right] \\
& =\left[\begin{array}{ccc}
B_{x} & 0 \\
C_{x} & 0 \\
C_{0}\left[\left(A_{x}-1\right) \tan \theta_{x}-C_{x}\left(x_{0}+L \sin \theta_{x}\right)\right] & \beta_{0}\left[B_{x} \tan \theta_{x}-D_{x} L \sin \theta_{x}+\left(1-D_{x}\right) x_{0}\right] & 1
\end{array}\right] .
\end{aligned}
$$

respectively. Equation (52) was previously derived. ${ }^{25}$ From Eqs. (52) and (53) it follows that a calibrated Gaussian aperture may be used to obtain a beam's phase front curvature and spot size by merely measuring beam positions and slopes.

When $C_{x}=0$, the Gaussian beam's spot size and radius of curvature remain unchanged and Eqs. (48) and (51) take on simpler forms:

$$
\begin{aligned}
& d_{x a 2}=d_{x a 1}-\frac{G_{x i}}{Q_{x 1 i}}-\frac{H_{x r}}{\beta_{0}}-\frac{Q_{x 1 r}}{Q_{x 1 i}} \frac{H_{x i}}{\beta_{0}} \\
& d_{x a 2}^{\prime}=d_{x a 1}^{\prime}+\frac{G_{x r}}{\beta_{0}}-\frac{Q_{x 1 r}}{Q_{x 1 i}} \frac{G_{x i}}{\beta_{0}}-\left(1+\frac{Q_{x 1 r}{ }^{2}}{Q_{x 1 i}{ }^{2}}\right) \frac{Q_{x 1 i} H_{x i}}{\beta_{0}^{2}}
\end{aligned}
$$

If $G_{x}$ is real and $H_{x}=0$, then the slope is changed while the position remains unaltered. This slope change may occur because of the presence of an optical element or because of a redefinition of the optic axis. Similarly, if $H_{x}$ is real and $G_{x}=0$, then the position is altered while the slope remains unchanged. These operations may be written as

$$
\begin{aligned}
d_{x a 2} & =d_{x a 1}+x_{0}, \\
d_{x a 2}^{\prime} & =d_{x a 1}^{\prime}+\tan \theta_{x},
\end{aligned}
$$

where the displacement of the optical element, $x_{0}$, and the slope of the optical element, $\tan \theta_{x}$, are strictly real. One may combine these two operations into a single matrix, using Eqs. (54) and (55) with real $G_{x}$ and $H_{x}$, and the generalized beam matrix for this optical element is
This result, along with the axis transformation matrix (60), is included in Table 1. The above matrix allows for the analysis of paraxially tilted complex optical systems. For example, the Gaussian beam matrix for a spherical mirror with curvature $R$ has $A_{x}=D_{x}=1, B_{x}=0, C_{x}=$ $-2 / R$, and $L=0$. From Eq. (60) the generalized beam matrix is

$$
T_{\text {spherical mirror }, x}=\left[\begin{array}{ccc}
1 & 0 & 0 \\
-2 R^{-1} & 1 & 0 \\
2 \beta_{0} x_{0} R^{-1} & 0 & 1
\end{array}\right] .
$$

\section{B. Linearly Profiled Optical Elements}

Generalized beam matrix representations for linearly profiled optical elements are found in this subsection. While the thin prism and the exponential aperture are optical elements that are linearly profiled in complex phase, complex prismlike media are linearly profiled in complex propagation constant.

The generalized Gaussian beam matrix for the complex prismlike medium introduced here is given by Eq. (45). The next optical element considered is an aperture that has an exponential transmission or reflection profile. Since the exponential aperture is a thin optical element, the output is just the input multiplied by the exponential transmission function. For an exponential of damping length $w_{e a, x}$, tilted at the small angle $\theta_{x}$ and offset by the distance $x_{0}$, the output electric field is

$$
\begin{aligned}
\left\{E_{0}^{\prime} \exp [\right. & \left.\left.-i\left(Q_{x} x^{2} / 2+Q_{y} y^{2} / 2+S_{x} x+S_{y} y+P\right)\right]\right\}_{\text {out }} \\
= & \left\{E_{0}^{\prime} \exp \left[-i\left(Q_{x} x^{2} / 2+Q_{y} y^{2} / 2+S_{x} x+S_{y} y+P\right)\right]\right\}_{\text {in }} \\
& \times \exp \left[\left(x-x_{0}\right) /\left(w_{e a, x} \cos \theta_{x}\right)\right] .
\end{aligned}
$$


The output beam parameter, the displacement parameter, and the phase parameter may be found in terms of the input conditions by equating terms in Eq. (62):

$$
\begin{aligned}
Q_{x 2} & =Q_{x 1}, \\
S_{x 2} & =S_{x 1}+\frac{i}{w_{g a, x} \cos \theta_{x}}, \\
P_{2} & =P_{1}-\frac{i x_{0}}{w_{g a, x} \cos \theta_{x}} .
\end{aligned}
$$

The generalized beam matrix for the exponential aperture for the $x$ direction may now be obtained from Eqs. (43) and (44):

$$
T_{\text {exponential aperture }, x}=\left[\begin{array}{ccc}
1 & 0 & 0 \\
0 & 1 & 0 \\
i /\left(w_{e a, x} \cos \theta_{x}\right) & 0 & 1
\end{array}\right]
$$

By our postulate the exponential aperture varied in only the $x$ direction, and thus the generalized beam matrix in the $y$ direction is the identity matrix. However, if the axis of the aperture were not parallel to the $x$ direction, there would be a similar matrix for the $y$ direction.

As an intermediate step to determining the generalized beam matrix for a thin prism, the matrix for a tilted linear boundary between two homogeneous media is found. The accumulated phase of a plane wave after propagating a distance $z>z^{\prime}$, where $z^{\prime}$ is the position of a boundary between two media with propagation constants $k_{01}$ and $k_{02}$, is

$$
\begin{aligned}
E_{\mathrm{out}}^{\prime} & =E_{\mathrm{in}}^{\prime} \exp \left(-i k_{01} z^{\prime}\right) \exp \left[-i k_{02}\left(z-z^{\prime}\right)\right] \\
& =E_{\mathrm{in}}^{\prime} \exp \left(-i k_{02} z\right) \exp \left[-i\left(k_{01}-k_{02}\right) z^{\prime}\right]
\end{aligned}
$$

Here the boundary position is allowed to vary with transverse distance. The boundary under consideration is linear in the $x z$ plane and is defined as being displaced from the axis by an amount $x_{0}$. Thus

$$
z^{\prime}=\left(x-x_{0}\right) \tan \theta_{x}
$$

where $\theta_{x}$ is measured from the $x$ axis (toward the $z$ axis). Equation (69) may be combined with Eq. (68), and it follows that

$$
\begin{aligned}
&\left\{E_{0}^{\prime} \exp \left[-i\left(Q_{x} x^{2} / 2+Q_{y} y^{2} / 2+S_{x} x+S_{y} y+P\right)\right]\right\}_{\text {out }} \\
&=\left\{E_{0}^{\prime} \exp \left[-i\left(Q_{x} x^{2} / 2+Q_{y} y^{2} / 2+S_{x} x+S_{y} y+P\right)\right]\right\}_{\text {in }} \\
& \times \exp \left(-i k_{02} z\right) \exp \left[-i\left(k_{01}-k_{02}\right)\left(x-x_{0}\right) \tan \theta_{x}\right] .
\end{aligned}
$$

When we equate terms as above, the output phase parameter is

$$
P_{2}=P_{1}-\left(k_{01}-k_{02}\right) x_{0} \tan \theta_{x}
$$

and the generalized beam matrix in the $x$ direction is

$$
T_{\text {linear boundary, } x}=\left[\begin{array}{ccc}
1 & 0 & 0 \\
0 & k_{01} / k_{02} & 0 \\
\left(k_{01}-k_{02}\right) \tan \theta_{x} & 0 & 1
\end{array}\right] .
$$

The matrix for a simple prism may be obtained by com- bining two linear boundaries:

$$
T_{\text {thin prism }, x}=\left[\begin{array}{ccc}
1 & 0 & 0 \\
0 & 1 & 0 \\
\left(k_{0}-\beta_{\text {air }}\right)\left(\tan \theta_{x 2}-\tan \theta_{x 1}\right) & 0 & 1
\end{array}\right] .
$$

The thin prism is allowed to have gain or loss $\left(k_{0}=\beta_{0}+\right.$ $i \alpha_{0}$ ), as are several of the subsequent optical elements considered here. Thus an amplifier (or absorber) wedge, for example, is included in Eq. (73).

The final optical element considered in this subsection is the tilted (flat) mirror. The tilted mirror has the same characteristic as Eq. (57), and thus the generalized beam matrix is given by Eq. (58) with $x_{0}=0$. The results of this subsection are summarized in the first five rows of Table 2.

\section{Quadratically Profiled Optical Elements}

When $S_{x}=0$, the $3 \times 3$ matrix method reduces to the $2 \times 2$ method of Kogelnik. Thus, for elements in which $G_{x}=H_{x}=0$, the $A_{x}, B_{x}, C_{x}$, and $D_{x}$ elements are the same as those of conventional Gaussian beam optics. It is the purpose of this subsection to extend the range of validity of these conventional optical elements so that they may be displaced, misaligned, or curved.

The generalized beam matrix for a complex lenslike medium was derived above and is given in Eq. (40). The next optical element considered is an aperture that has a Gaussian transmission or reflection profile. For a Gaussian aperture with $1 / e$ amplitude width $w_{g a, x}$ tilted at an angle $\theta_{x}$ and offset by length $x_{0}$, the output electric field is

$$
\begin{aligned}
&\left\{E_{0}^{\prime} \exp \left[-i\left(Q_{x} x^{2} / 2+Q_{y} y^{2} / 2+S_{x} x+S_{y} y+P\right)\right]\right\}_{\text {out }} \\
&=\left\{E_{0}^{\prime} \exp \left[-i\left(Q_{x} x^{2} / 2+Q_{y} y^{2} / 2+S_{x} x+S_{y} y+P\right)\right]\right\}_{\text {in }} \\
& \times \exp \left[-\left(x-x_{0}\right)^{2} /\left(w_{g a, x}{ }^{2} \cos ^{2} \theta_{x}\right)\right] \exp \left(-y^{2} / w_{g a, y}{ }^{2}\right) .
\end{aligned}
$$

As in Subsection 3.B, the output beam parameter, the displacement parameter, and the phase parameter may be found in terms of the input conditions by equating terms in Eq. (74):

$$
\begin{aligned}
\frac{Q_{x 2}}{2} & =\frac{Q_{x 1}}{2}-\frac{i}{w_{g a, x^{2}} \cos ^{2} \theta_{x}}, \\
S_{x 2} & =S_{x 1}+i \frac{2 x_{0}}{w_{g a, x} \cos ^{2} \theta_{x}}, \\
P_{2} & =P_{1}-i \frac{x_{0}^{2}}{w_{g a, x} \cos ^{2} \theta_{x}}
\end{aligned}
$$

Without loss of generality, it may be assumed that the medium surrounding the aperture is completely characterized by the relation $k(x, y, z)=\beta_{\text {air }}$. Under this condition the generalized beam matrix may be obtained from Eqs. (43), (44), (75), and (76):

$$
T_{\text {Gaussian aperture, } x}=\left[\begin{array}{ccc}
1 & 0 & 0 \\
-2 i /\left(\beta_{\text {air }} w_{g a, x}^{2} \cos ^{2} \theta_{x}\right) & 1 & 0 \\
2 i x_{0} /\left(w_{g a, x}{ }^{2} \cos ^{2} \theta_{x}\right) & 0 & 1
\end{array}\right] .
$$

There is a similar matrix governing the $y$ distribution of the fields. 
Table 2. Generalized Beam Matrices for Profiled Elements Misaligned in the $x$ Direction

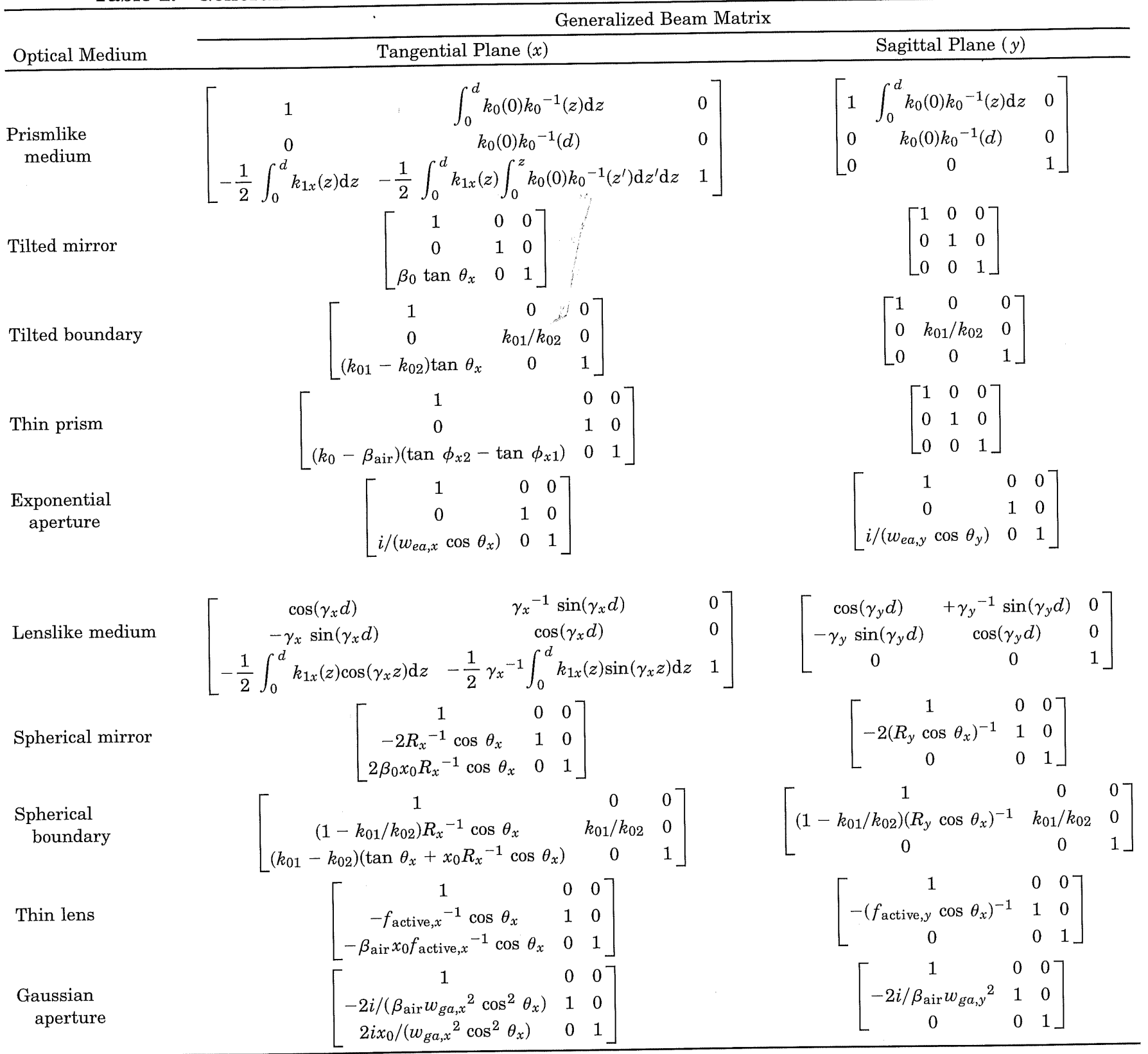

Just as in the linear case, Eq. (68) may be used to obtain the matrix representation of spatially nonlinear boundaries between complex media. In particular, the boundary of interest here is initially assumed to be spherical, so that

$$
x^{2}+y^{2}+\left(z^{\prime}+R\right)^{2}=R^{2} .
$$

This equation may be rewritten as

$$
z^{\prime}=-R\left[1-\left(1-\frac{x^{2}+y^{2}}{R^{2}}\right)^{1 / 2}\right] \text {. }
$$

Typically the extent of the beam is much smaller than the spherical radius of the boundary, and Eq. (80) can be written approximately as

$$
z^{\prime} \approx-\frac{x^{2}}{2 R_{x}}-\frac{y^{2}}{2 R_{y}},
$$

where the boundary is also allowed to be approximately paraboloidal. In writing Eq. (79), we have assumed that the boundary was aligned perpendicular to the axis. More generally, it may be assumed that the boundary is rotated in the $x z$ plane and translated with respect to $x$. In this case an alternative coordinate system is of interest:

$$
\left(\begin{array}{l}
z^{\prime} \\
x^{\prime} \\
y^{\prime} \\
1
\end{array}\right)=\left[\begin{array}{cccc}
1 & 0 & 0 & 0 \\
0 & 1 & 0 & x_{0} \\
0 & 0 & 1 & 0 \\
0 & 0 & 0 & 1
\end{array}\right]\left[\begin{array}{cccc}
\cos \theta_{x} & \sin \theta_{x} & 0 & 0 \\
-\sin \theta_{x} & \cos \theta_{x} & 0 & 0 \\
0 & 0 & 1 & 0 \\
0 & 0 & 0 & 1
\end{array}\right]\left(\begin{array}{c}
z^{\prime \prime} \\
x^{\prime \prime} \\
y^{\prime \prime} \\
1
\end{array}\right),
$$

$$
\left(\begin{array}{c}
z^{\prime \prime} \\
x^{\prime \prime} \\
y^{\prime \prime} \\
1
\end{array}\right)=\left[\begin{array}{cccc}
\cos \theta_{x} & -\sin \theta_{x} & 0 & x_{0} \sin \theta_{x} \\
+\sin \theta_{x} & \cos \theta_{x} & 0 & -x_{0} \cos \theta_{x} \\
0 & 0 & 1 & 0 \\
0 & 0 & 0 & 1
\end{array}\right]\left(\begin{array}{l}
z^{\prime} \\
x^{\prime} \\
y^{\prime} \\
1
\end{array}\right) .
$$


In this coordinate system the boundary is aligned, and thus

$$
z^{\prime \prime}=-\frac{x^{\prime \prime 2}}{2 R_{x}}-\frac{y^{\prime \prime 2}}{2 R_{y}}
$$

Combining Eqs. (81), (83), and (84) results in

$$
\begin{aligned}
z^{\prime}= & -\frac{x^{2}}{2 R_{x}} \cos \theta_{x}+\left(\tan \theta_{x}+\frac{x_{0} \cos \theta_{x}}{R_{x}}\right) x \\
& -\frac{y^{2}}{2 R_{y} \cos \theta_{x}}-\left(\frac{x_{0}^{2} \cos \theta_{x}}{2 R_{x}}+x_{0} \tan \theta_{x}\right),
\end{aligned}
$$

where the approximation

$$
\left|\frac{\left(x-x_{0}\right) \sin \theta_{x}}{R_{x}}\right| \ll 1
$$

has been used to avoid twisting of the beam and higherorder aberrations. Now Eq. (85) may be combined with Eq. (68), and after some algebra it follows that

$$
\begin{aligned}
& T_{\text {spherical boundary }, x} \\
& \quad=\left[\begin{array}{ccc}
1 & 0 & 0 \\
\left(1-k_{01} / k_{02}\right) R_{x}{ }^{-1} \cos \theta_{x} & k_{01} / k_{02} & 0 \\
\left(k_{01}-k_{02}\right)\left(\tan \theta_{x}+x_{0} R_{x}{ }^{-1} \cos \theta_{x}\right) & 0 & 1
\end{array}\right],
\end{aligned}
$$

$T_{\text {spherical boundary, } y}$

$$
\begin{aligned}
= & {\left[\begin{array}{ccc}
1 & 0 & 0 \\
\left(1-k_{01} / k_{02}\right)\left(R_{y} \cos \theta_{x}\right)^{-1} & k_{01} / k_{02} & 0 \\
0 & 0 & 1
\end{array}\right], } \\
P_{2} & =P_{1}-x_{0}{ }^{2} \frac{\cos \theta x}{2 R_{x}}-x_{0} \tan \theta_{x} .
\end{aligned}
$$

The beam matrices for a thin lens may be derived by combining two spherical boundary matrices in the usual way, and it follows that

$$
\begin{aligned}
T_{\text {thin lens }, x}= & {\left[\begin{array}{ccc}
1 & 0 & 0 \\
-f_{\text {active }, x}{ }^{-1} \cos \theta_{x} & 1 & 0 \\
-\beta_{0} x_{0} f_{\text {active }, x}{ }^{-1} \cos \theta_{x} & 0 & 1
\end{array}\right], } \\
T_{\text {thin lens }, y}= & {\left[\begin{array}{ccc}
1 & 0 & 0 \\
-\left(f_{\text {active }, x} \cos \theta_{x}\right)^{-1} & 1 & 0 \\
0 & 0 & 1
\end{array}\right], }
\end{aligned}
$$

where

$$
f_{\text {active }, x} \equiv\left(1-\beta_{\text {air }}{ }^{-1} k_{0, \text { lens }}\right)\left(R_{x 1}{ }^{-1}-R_{x 2}{ }^{-1}\right) .
$$

The quantity $k_{0, \text { lens }}=2 \pi n_{0, \text { lens }} \lambda^{-1}+i \alpha_{\text {lens }}$ is the complex propagation constant of the lens material, and it may be noted that $f_{\text {active }, x}$ is therefore complex. When the lens is made of a lossless dielectric or when the low gain (or loss) per wavelength approximation is made, $f_{\text {active }, x}$ becomes the usual focal length $f_{x}$. The results of this subsection are summarized in the last five rows of Table 2.

\section{DISCUSSION}

With the results from Section 3, one may propagate a Gaussian beam's spot size, phase front curvature, position, and slope through an optical system in which the elements may be misaligned and nonlossless. The general procedure is begun by finding the input beam parameters $Q_{x}$ and $Q_{y}$ from Eq. (19) and the input displacement parameters $S_{x}$ and $S_{y}$ from Eq. (24). However, in the usual case, the beam is input in a homogeneous medium such as free space, and the much simpler Eq. (25) may be used instead of Eq. (24). The system matrix may be obtained by multiplying the matrix representation for each of the optical elements in the reverse of the order in which they are encountered by the beam. These matrix representations are given in Tables 1 and 2 . Once the system matrix and the input beam parameters have been obtained, the output beam parameters may be found from the Kogelnik transformation [Eq. (43)]. Similarly, the output displacement parameters may be obtained from Eq. (44). From the output beam parameters the output spot size and phase curvature may be found from Eq. (19). Finally, the beam's output position and slope may be obtained from Eq. (25).

The above procedure may be made more transparent for those familiar with Gaussian beam matrix theory if the following analogies are noted:

$$
\begin{aligned}
& Q_{x} \leftrightarrow S_{x} \\
& Q_{x}=\frac{\beta_{0}}{R_{x}}-i \frac{2}{w_{x}{ }^{2}} \leftrightarrow S_{x}=-Q_{x} d_{x a}+\beta_{0} d_{x a}^{\prime} \\
& \frac{Q_{x 2}}{k_{02}}=\frac{C_{x}+D_{x} Q_{x 1} / k_{01}}{A_{x}+B_{x} Q_{x 1} / k_{01}} \leftrightarrow S_{x 2}=\frac{S_{x 1}}{A_{x}+B_{x} Q_{x 1} / k_{01}} \\
& +\frac{G_{x}+H_{x} Q_{x 1} / k_{01}}{A_{x}+B_{x} Q_{x 1} / k_{01}}
\end{aligned}
$$

Thin lens $\leftrightarrow$ Thin prism

$$
\begin{gathered}
\text { Gaussian aperture } \leftrightarrow \text { Exponential aperture } \\
\text { Spherical mirror } \leftrightarrow \text { Tilted flat mirror }
\end{gathered}
$$

Complex lenslike medium $\leftrightarrow$ Complex prismlike medium

There is no analytical matrix representation for a complex lenslike medium that is arbitrarily tapered and curved. However, it is possible to obtain the matrix representation for complex lenslike media with several specific tapering and curvature functions. To obtain the matrix representation for such an optical element, one must first find the complete complex propagation constant. In general, the curvature of the gain axes [Eqs. (10) and (11)] may be different from the curvature of the index-of-refraction axes [Eqs. (8) and (9)]. In any case, Eq. (7) is useful for obtaining the propagation constant. Once the propagation constant is known, differential equations (27) and (28) must be solved. If exact analytic solutions ${ }^{26,27}$ are not readily obtained, WKB approximation techniques, ${ }^{26}$ solution-generating methods ${ }^{27}$ series expansions, piecewise approximation techniques, ${ }^{28}$ propagation constant approximations, ${ }^{28}$ or numerical methods ${ }^{29}$ may be used. The solutions to Eq. (27) must be put into the form of Eq. (29) to yield $A_{x}, A_{y}, B_{x}$, and $B_{y}$. The elements $C_{x}$, 
$C_{y}, D_{x}$, and $D_{y}$ may then be obtained from the relationships $C=\mathrm{d} A / \mathrm{d} z, D=\mathrm{d} B / \mathrm{d} z$. The remaining elements, $G_{x}, G_{y}, H_{x}$, and $H_{y}$, are obtained from the integrals in Eqs. (35) and (36).

The matrices in Tables 1 and 2 are also generalized from previous similar tables of beam matrices in that the possibility of media with high nonsaturating gain per wavelength is included. Thus the complex propagation constant $k_{0}\left(k_{0}=2 \pi n / \lambda+i \alpha_{0}\right)$ is employed instead of the more commonly used index of refraction $n$.

As an example of the formalism, the transmission characteristics of an exponential aperture having the matrix shown in Table 2 are examined. Because of the simplicity of the matrix, Eqs. (19) and (25) may be combined into Eqs. (54) and (55), which immediately reduce to

$$
\begin{aligned}
d_{x a 2} & =d_{x a 1}+\frac{w_{x 1}^{2}}{2 w_{e a, x}}, \\
d_{x a 2}{ }^{\prime} & =d_{x a 1}{ }^{\prime}+\frac{w_{x 1}{ }^{2}}{2 R_{x 1} w_{e a, x}},
\end{aligned}
$$

where $w_{e a, x}$ is the damping width of the exponential aperture. A beam's spot size and phase curvature are unaffected by the aperture. Just as for the Gaussian aperture, one may use the exponential aperture for beam diagnostics by measuring only beam positions and slopes.

\section{CONCLUSION}

All Gaussian beam optical systems are, to some extent, nonlossless. Similarly, tilts and misalignments within the system are inevitable. Furthermore, in modern optical systems design these effects are often not accidental but are introduced for system enhancement. For example, high diffraction loss (unstable) resonators use transverse losses to achieve high power in a single highly stable mode, and prism pairs are used for chirp compensation. It is therefore useful to have a systematic methodology to analyze these systems.

A novel transfer-matrix formalism has been developed from first principles to propagate Gaussian beams of light in misaligned, nonlossless optical systems. The optical systems are represented by complex $3 \times 3$ generalized Gaussian beam matrices, and matrices have been developed for several optical elements. Analogies have been used for nomenclature and have suggested new optical elements such as the exponential variable-reflectivity mirror and the complex prismlike medium.

\section{APPENDIX A: REDUCTION TO RAY MATRICES IN LOSSLESS OPTICAL SYSTEMS}

The purpose of this appendix is to explore the relationship between generalized beam matrices and generalized ray matrices for fundamental Gaussian beam propagation.

The Kogelnik transformation and the displacement transformation may be written as

$$
\begin{aligned}
\frac{Q_{x 2}}{k_{02}} & \equiv \frac{C_{x}+D_{x} Q_{x 1} / k_{01}}{A_{x}+B_{x} Q_{x 1} / k_{01}}, \\
S_{x 2} & =\frac{S_{x 1}}{A_{x}+B_{x} Q_{x 1} / k_{01}}+\frac{G_{x}+H_{x} Q_{x 1} / k_{01}}{A_{x}+B_{x} Q_{x 1} / k_{01}},
\end{aligned}
$$

where, in general, the matrix elements are complex.
However, in the paraxial ray matrix theory the matrix elements are real, and this restriction will be used here. Furthermore, the medium surrounding the optical system will be assumed to be lossless and homogeneous. Thus Eqs. (A1) and (A2) reduce to

$$
\begin{aligned}
\frac{Q_{x 2}}{\beta_{0}} & =\frac{\left(C_{x}+D_{x} Q_{x 1 r} / \beta_{0}\right)+i\left(D_{x} Q_{x 1 i} / \beta_{0}\right)}{\left(A_{x}+B_{x} Q_{x 1 r} / \beta_{0}\right)+i\left(B_{x} Q_{x 1 i} / \beta_{0}\right)}, \\
S_{2 x} & =\frac{\left(S_{x 1 r}+G_{x}+H_{x} Q_{x 1 r} / \beta_{0}\right)+i\left(S_{x 1 i}+H_{x} Q_{x 1 i} / \beta_{0}\right)}{\left(A_{x}+B_{x} Q_{x 1 r} / \beta_{0}\right)+i\left(B_{x} Q_{x 1 i} / \beta_{0}\right)},
\end{aligned}
$$

where each of the quantities in parentheses is real. If we take the real and imaginary parts of these equations and use the relations

$$
\begin{aligned}
& d_{x a 2}=-\frac{S_{x 2 i}}{Q_{x 2 i}} \\
& d_{x p 2}=-\frac{S_{x 2 r}}{Q_{x 2 r}}, \\
& d_{x a 2}^{\prime}=\frac{Q_{x r 2}}{\beta_{0}}\left(d_{x a 2}-d_{x p 2}\right),
\end{aligned}
$$

it follows after some algebra that

$$
d_{x a 2}=A_{x} d_{x a 1}+B_{x} d_{x a 1}^{\prime}+\frac{B_{x} G_{x}-A_{x} H_{x}}{\beta_{0}} .
$$

Taking a $z$ derivative of this equation and making use of the definitions of $G_{x}$ [Eq. (35)] and $H_{x}$ [Eq. (36)], we can see that

$$
d_{x a 2}^{\prime}=C_{x} d_{x a 1}+D_{x} d_{x a 1}^{\prime}+\frac{D_{x} G_{x}-C_{x} H_{x}}{\beta_{0}} .
$$

These equations may be put into ray matrix form as

$$
\left(\begin{array}{c}
d_{x a 2} \\
d_{x a 2}^{\prime} \\
1
\end{array}\right)=\left[\begin{array}{ccc}
A_{x} & B_{x} & E_{x} \\
C_{x} & D_{x} & F_{x} \\
0 & 0 & 1
\end{array}\right]\left(\begin{array}{c}
d_{x a 1} \\
d_{x a 1}^{\prime} \\
1
\end{array}\right),
$$

where

$$
\begin{aligned}
& E_{x}=\left(B_{x} G_{x}-A_{x} H_{x}\right) / \beta_{0}, \\
& F_{x}=\left(D_{x} G_{x}-C_{x} H_{x}\right) / \beta_{0} .
\end{aligned}
$$

Equation (A10) is a conventional generalized ray matrix. Thus the relationship between the ray formalism and the generalized beam matrix formalism is identified in Eqs. (A11) and (A12). For purposes of reference, Eqs. (A11) and (A12) may be inverted as

$$
\begin{aligned}
& G_{x}=\beta_{0}\left(A_{x} F_{x}-C_{x} E_{x}\right), \\
& H_{x}=\beta_{0}\left(B_{x} F_{x}-D_{x} E_{x}\right),
\end{aligned}
$$

where the unimodularity condition has been assumed.

If the matrix element definitions [Eqs. (35) and (36)] are used along with the definition of the displacement of the refraction index axis [Eq. (8)], then the ray matrix elements [Eqs. (A11) and (A12)] for a curved real lenslike medium are

$$
\begin{aligned}
& E_{x}=\gamma_{x} \int_{0}^{z} d_{\beta x}(z) \sin \left[\gamma_{x}\left(z-z^{\prime}\right)\right] \mathrm{d} z^{\prime}, \\
& F_{x}=\gamma_{x} \int_{0}^{z} d_{\beta x}(z) \cos \left[\gamma_{x}\left(z-z^{\prime}\right)\right] \mathrm{d} z^{\prime},
\end{aligned}
$$


where

$$
\gamma_{x} \equiv\left(\frac{n_{2 x}}{n_{0}}\right)^{1 / 2}
$$

This special case result [Eqs. (A15)-(A17)] is in agreement with that of Hardy. ${ }^{5}$

\section{ACKNOWLEDGMENT}

This work was supported in part by the National Science Foundation under grant ECS-9014481.

\section{REFERENCES}

1. A. Gerrard and J. M. Burch, Introduction to Matrix Methods in Optics (Wiley, New York, 1975), pp. 24-26.

2. A. Cayley, "A memoir on the theory of matrices," Philos. Trans. R. Soc. London CXLVIII, 17-37 (1858); also published in The Collected Mathematical Papers of Arthur Cayley (Cambridge U. Press, Cambridge, 1889), Vol. 2, pp. $475-496$.

3. K. Halbach, "Matrix representation of Gaussian optics," Am. J. Phys. 32, 90-108 (1964).

4. J. A. Arnaud, "Degenerate optical cavities. II. Effects of misalignments," Appl. Opt. 8, 1909-1917 (1969).

5. A. Hardy, "Beam propagation through parabolic-index waveguides with distorted optical axis," Appl. Phys. 18, 223226 (1979).

6. A. E. Siegman, Lasers (University Science, Mill Valley, Calif., 1986), pp. 607-616.

7. H. Kogelnik, "Imaging of optical modes-resonators with internal lenses," Bell Syst. Tech. J. 44, 455-494 (1965).

8. P. K. Tien, J. P. Gordon, and J. R. Whinnery, "Focusing of a light beam of Gaussian field distribution in continuous and periodic lens-like media," IEEE Proc. 53, 129-136 (1965).

9. L. W. Casperson, "Gaussian light beams in inhomogeneous media," Appl. Opt. 12, 2434-2441 (1973).

10. L. W. Casperson, "Mode stability of lasers and periodic optical systems," IEEE J. Quantum Electron. QE-10, 629-634 (1974).

11. M. Nazarathy, A. Hardy, and J. Shamir, "Generalized mode propagation in first-order optical systems with loss or gain," J. Opt. Soc. Am. 72, 1409-1420 (1982).

12. A. A. Tovar and L. W. Casperson, "Off-axis complex- argument polynomial-Gaussian beams in optical systems," J. Opt. Soc. Am. A 8, 60-68 (1991).

13. N. McCarthy and P. Lavigne, "Optical resonators with Gaussian reflectivity mirrors: misalignment sensitivity," Appl. Opt. 22, 2704-2708 (1983).

14. N. McCarthy and M. Morin, "High-order transverse modes of misaligned laser resonators with Gaussian reflectivity mirrors," Appl. Opt. 28, 2189-2191 (1989).

15. D. Marcuse, "The effect of the $\nabla n^{2}$ term on the modes of an optical square-law medium," IEEE J. Quantum Electron. QE-9, 958-960 (1973).

16. C. Yeh, L. W. Casperson, and W. P. Brown, "Scalar-wave approach for single-mode inhomogeneous fiber problems," Appl. Phys. Lett. 34, 460-462 (1979).

17. J. D. Love, C. D. Hussey, A. W. Snyder, and R. A. Sammut, "Polarization corrections to mode propagation on weakly guiding fibers," J. Opt. Soc. Am. 72, 1583-1591 (1982).

18. C. C. Su, "On the scalar approximation in fiber optics," IEEE Trans. Microwave Theory Tech. 36, 1100-1103 (1988).

19. R. C. Jones, "A new calculus for the treatment of optical systems. V. A more general formulation and description of another calculus," J. Opt. Soc. Am. 37, 107-110 (1947).

20. A. A. Tovar and L. W. Casperson, "Gaussian beam optical systems with high gain or high loss media," IEEE Trans. Microwave Theory Tech. (to be published).

21. W. R. Bennett, Appl. Opt. Suppl. 2, 3-33 (1965).

22. B. N. Perry, P. Rabinowitz, and M. Newstein, "Wave propagation in media with focused gain," Phys. Rev. A 27, 1989-2002 (1983).

23. L. W. Casperson, "Beam modes in complex lenslike media and resonators," J. Opt. Soc. Am. 66, 1373-1379 (1976).

24. L. W. Casperson and S. J. Sheldrake, "Beam deflection and isolation in laser amplifiers," Opt. Commun. 12, 349-353 (1974).

25. L. W. Casperson and S. Lunnam, "Gaussian modes in high loss laser resonators," Appl. Opt. 14, 1193-1199 (1975).

26. L. W. Casperson, "Beam propagation in tapered quadraticindex waveguides: analytical solutions," J. Lightwave Technol. LT-3, 264-272 (1985).

27. A. A. Tovar and L. W. Casperson, "Beam propagation in parabolically tapered graded-index waveguides," Appl. Opt. 33, 7733-7739 (1994).

28. L. W. Casperson, "Beam propagation in periodic quadraticindex waveguides," Appl. Opt. 24, 4395-4403 (1985).

29. L. W. Casperson and J. L. Kirkwood, "Beam propagation in tapered quadratic-index waveguides: numerical solutions," J. Lightwave Technol. LT-3, 256-263 (1985). 